\title{
Ion Desolvation in Microporous Electrodes with Liquid Electrolytes
}

\author{
Leying Qing ${ }^{1}$, Ting Long ${ }^{1}$, Hongping $\mathrm{Yu}^{1}, \mathrm{Yu} \mathrm{Li}^{1}$, Weiqiang Tang${ }^{1}$, Bo Bao ${ }^{1}$, and \\ Shuangliang Zhao ${ }^{1}$ \\ ${ }^{1}$ East China University of Science and Technology
}

October 28, 2020

\begin{abstract}
A deep understanding of ion desolvation in microporous electrodes is helpful for achieving efficient energy storage. Herein, we evaluate the contribution of ion desolvation to electrochemical performance of microporous electrodes with a proposed multiscale approach. By integrating the molecular version with the simple version of classical density functional theory, we first determine the solvation diameters of ions in confined molecular solvent, and then predict the capacitances of microporous electrodes through a solvation-diameter-dependent coarse-grained model. We find that the solvation diameter displays an oscillatory decline as decreasing the pore size of nanoslit, and upon this relation in combination with the pore size distribution of microporous electrode we give satisfactory predictions of the capacitances of practical electrodes compared with experimental measurements. This work not only provides a feasible multiscale tool for predicting the capacitances of microporous electrodes involving liquid electrolytes, but also casts insights for the design and preparation of high-performance supercapacitors.
\end{abstract}

\section{Introduction}

Growing energy demand and environmental protection inspire the exploit of renewable energy such as solar energy, wind energy and ocean energy. Its intermittence necessitates the development of high-performance storage devices to achieve excellent energy storage and thus stabilize the large-scale practical supplies in the form of electrochemical energy. ${ }^{1}$ Supercapacitors (also called ultracapacitors) and batteries are important candidates for electrochemical energy storages, ${ }^{2}$ and have been extensively studied by experiments and theoretical methods. ${ }^{3-5}$ The physical adsorption and diffusion of electrolytes in supercapacitor ensure its high power density ${ }^{6-7}$ and outstanding cycling performance. ${ }^{8}$ However, the energy storage density is much lower compared to the chemical storage batteries. ${ }^{\mathbf{9}}$

In recent years, the microporous materials involving liquid electrolytes have exhibited tremendous potential for promoting the energy density of supercapacitor. Gogotsi and his co-workers et al. ${ }^{\mathbf{1 0} \mathbf{1 1}}$ manufactured a microporous $\mathrm{TiC}$ carbide-derived carbon (TiC-CDC) electrode, and this electrode displays outstanding performance in energy storage capacity. For example, by tuning the average pore size of the microporous electrode to $1.0 \mathrm{~nm}$, Chmiola et al. ${ }^{\mathbf{1 2}}$ found that the capacitance anomalously increases for the supercapacitor involving acetonitrile-based liquid electrolyte. Further decreasing the pore size down to about one ion size, the anomalously promoted capacitance is observed. ${ }^{13}$ These results are soon confirmed by different theoretical studies such as Monte Carlo simulations, ${ }^{\mathbf{1 4}}$ molecular dynamics (MD) simulations, ${ }^{\mathbf{1 5}-16}$ atomistic MD simulations, ${ }^{17-18}$ and theoretical methods. ${ }^{19-20}$

The mechanism for the abnormally increased capacitance can be generally attributed to two aspects: the confinement effect (i.e., the steric effect of ions and the overlapping effect of electrostatic potentials) and the ion desolvation. ${ }^{\mathbf{2 1 - 2 2}}$ Specifically, for the confinement effect in nanoscale pores, both the overlapped electrostatic potential $^{\mathbf{2 3}}$ and the convex inner surfaces of electrodes ${ }^{\mathbf{2 4 - 2 5}}$ contribute to the high-performance of supercapacitors. The ion desolvation is closely associated with the ion adsorption amount. ${ }^{\mathbf{2 6 - 2 7}}$ By integrating the ion desolvation with hierarchical nanopores, the microporous electrodes have attracted considerable interests 
to achieve excellent charge storage efficiency in recent years. ${ }^{\mathbf{2 8 - 2 9}}$ However, because the ion desolvation, usually occurred in confined space, causes the rearrangement of microscopic solvent structure surrounding the ion, leading to the variations of solvent local density and ion solvation diameter, ${ }^{30-31}$ the relation between the solvation diameter of ion in nanopore with the pore size is highly desirable yet lacking, and this causes a significant bottleneck for properly understanding the contribution of ion desolvation to the capacitance of microporous electrodes. Compromisingly, unified ion size is employed in most coarse-grained studies on electrochemical properties of confined electrolytes disregarding the variation of pore size. ${ }^{\mathbf{2 0}, \mathbf{3 2}}$

Theoretical methods and computer simulations are employed to investigate the ion solvation in bulk solutions and interfacial systems on molecular level. ${ }^{33-35}$ Recently we proposed a model to examine the ion desolvation in confined water by incorporating the molecular density functional theory (MDFT) with an proposed desolvation model, ${ }^{36}$ and successfully demonstrated the disturbed hydration shell and the decreased hydration numbers of ions in nanoslits. MDFT is a molecular version of the classical density functional theory (CDFT), which additionally involves the molecular orientation as variables in local density distribution. ${ }^{\mathbf{3 7}}$ Herein, we integrate a similar desolvation model with coarse-grained description of solvated ions in nanopores to investigate the ion desolvation effect on capacitance. Particularly, we evaluate the capacitance by adopting the simple version of CDFT, in which the ionic components are described with coarse-grained spherical particles. Historically this version is simply called as CDFT. CDFT has been applied to predict the capacitances by accurately accounting for the excluded volume effect and electrostatic correlation in confined simple electrolytes. ${ }^{\mathbf{3 8 - 4 0}}$

By combining the MDFT and CDFT, the ion desolvation and its contribution to the capacitance of the microporous electrodes are investigated. We show that this combination can unravel the ion desolvation effect to electrochemical properties, and give satisfactory predictions on the capacitances of practical microporous electrodes with liquid electrolytes as long as the pore size distribution (PSD) is provided.

The remainder of this work is organized as follows. In Section 2, the ion desolvation in the microporous electrode is introduced and the essential equations of MDFT and CDFT are provided. In Section 3, the solvation diameters of ions in different nanoslits under ambient condition are evaluated by using the MDFT, and thereafter the capacitances of the nanoslits with different pore sizes are predicted by using CDFT. Next, integrating the pore-size dependent capacitances with the PSD, we study the ion desolvation contribution to the overall capacitance of the microporous electrodes in comparison with experimental measurements. Finally, a brief summary is given in Section 4.

\section{Molecular Model and Theory}

\subsection{Ion Desolvation in Nanoslit}

A positively charged microporous TiC-CDC electrode with specific PSD is depicted in Figure 1 . The PSD represents the probability distribution of the nanopores with different pore sizes embedded in the microporous electrode. Here we model each nanopore with nanoslit composed of two parallel charged flat surfaces. The measured PSD of the microporous electrode material prepared by Chmiola et al ${ }^{\mathbf{1 2}}$ is depicted in Figure 1(a) , indicating that the pore widths of most pores are between $0.5 \mathrm{~nm}$ and $0.7 \mathrm{~nm}$, and this clearly states that the nanoscale pores are dominant for determining the electrochemical properties of microporous electrodes. In most cases, the ions solvated in liquid electrolytes need to remove their solvation shells in order to penetrate into tiny pores. This process is conventionally referred to as ion desolvation, which can be often observed when ions transport through bio-channels. ${ }^{41}$ 

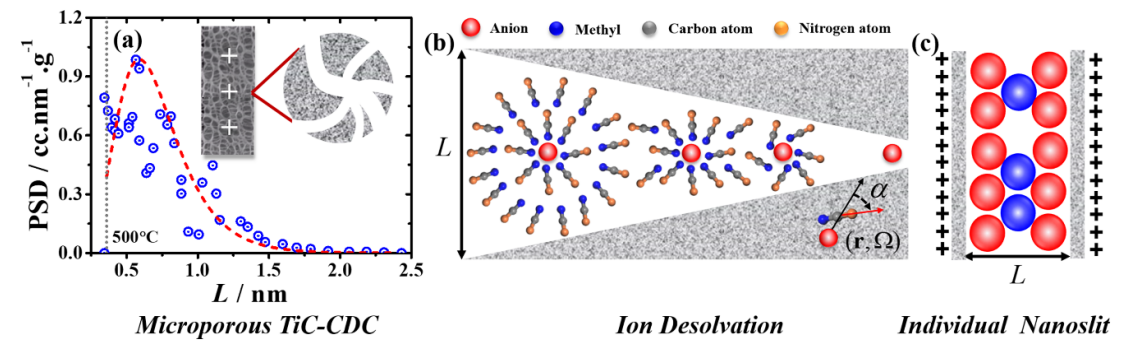

Figure 1 . (a) Schematic diagram of microporous TiC-CDC electrode with specific PSD obtained from ref. 12. (b) Illustration of ion desolvation caused by geometrical confinement of nanoslit. (c) A representative nanoslit in the positively charged electrode.

Figure 1 (b) displays the schematic graph of ion desolvation in confined liquid, in which the anion is encircled by solvent molecules due to the static electronic attraction. Here, acetonitrile (MeCN) solvent is considered in order to perform a faithful comparison with experiments. MeCN is a typical linear polar fluid and it can be modeled as a linear molecule containing three parts ${ }^{\mathbf{4 2}}$ : the methyl group (blue sphere), the carbon group (grey sphere), and the nitrogen group (yellow sphere). The solvent structure of MeCN surrounding the solute ion is described by the local density distribution of MeCN molecules, , which depends on both the spatial position, , and the Euler angle, , of MeCN molecule in space.

The spatial orientation of a MeCN molecule can be alternatively characterized by the included angle between the linear MeCN molecule and the vector connecting the solute ion to the carbon group of MeCN, designated as . In this work, the cosine value of , i.e .,, is employed to describe the spatial orientation of MeCN, which can be converted from the Euler angle through a rotation matrix operation. ${ }^{43}$ In nanopores, the interfacial interaction and the geometrical confinement effect may destroy the solvation structure of ion, leading to the rearrangement of local density distribution of $\mathrm{MeCN}$, and this feature becomes more pronounced as the pore size decreases down to several molecule sizes, resulting in a significant decrease of the MeCN number within the first solvation shell. When further decreasing the pore size, the solvation diameter of ion will decrease until the occurrence of a bare ion.

Since not all solvent molecules within the first solvation shell contribute to the solvation diameter of ions, ${ }^{\mathbf{4 4}-45}$ the spatial orientation distribution of MeCN can be additionally introduced to distinguish the contributing molecules. This idea has been applied to evaluate the ion hydration size in confined liquid water by using the MDFT, in which of the contributing water molecules in the first solvation shell was determined as $[-1.0,-0.44]$ for cation and $[0.48,1.0]$ for anion. ${ }^{36}$ In this work, we adopt the same concept to calculate the solvation diameter of ions in confined $\mathrm{MeCN}$ :

, (1)

\section{Hosted file}

image12.wmf available at https://authorea.com/users/370711/articles/489294-ion-desolvationin-microporous-electrodes-with-liquid-electrolytes

where is the volume of the solvated ion, i.e .,, with being the solvation diameter. is the volume of the bare ion, and its diameter, , is evaluated from the Barker-Henderson theory. ${ }^{\mathbf{4 6}}$ represents the occupied volume of contributing MeCN molecules, and being estimated through . Here is the number density of bulk MeCN solvent, and is the solvation number of MeCN. For an anion, the solvation number is calculated through the following expression:

,$(2)$

where with, and is the critical orientation angle in the first solvation shell to weight the contribution of $\mathrm{MeCN}$ molecules to the solvation diameter ${ }^{\mathbf{3 6}}$ is the local orientation-dependent distribution function of 
MeCN solvent surrounding the anion. represents the radius of the first valley in . In general, the value of varies at different orientations. In other words, can be expressed as a function of ,i.e .,. The solvation diameter can be solved by combining eqs. (1) and (2) once the critical orientation angle is determined.

We determine with the help of MDFT. Within the framework of MDFT, the ion solvation in MeCN solvent can be described in the grand canonical ensemble, in which the solvent chemical potential, the system volume , and the system temperature are fixed. A single ion is immersed in MeCN under given thermodynamic condition, and by minimizing the grand potential functional of the inhomogeneous solvent system, the local density distribution of $\mathrm{MeCN}$, can be obtained. ${ }^{\mathbf{4 7}}$ The grand potential functional is associated with the solvation free energy functional, , through:

,$(3)$

where represents the grand potential of the reference bulk solvent. In general, includes three contributions: the ideal term, the external contribution, and the excess term due to the intermolecular interactions.$^{\mathbf{3 7}}$ The expression of the solvation free energy functional follows:

Here, denotes the deviation of local density with respect to the bulk one, and is the Boltzmann constant. is the angular-dependent direct correlation function (DCF) of the bulk solvent. Here DCF is prepared in prior by solving the molecular Orenstein-Zernike equation with the help of exact pair correlation function extracted from simulation. ${ }^{48}$ represents the external potential consisting the solute-solvent interaction and the interfacial interaction. The former includes the pairwise Lennard-Jones (LJ) potential energy and direct Coulomb interaction between the solute ion and MeCN. The latter is the non-electrostatic interaction between the MeCN solvent and flat walls, and it can be described by the $10-4$ potential. ${ }^{49}$ For ion dissolved in the solvent free of confinement, the latter interaction vanishes and only the solute-solvent interaction is involved. The detailed expressions of are given in Supporting Information (SI) .

\section{2 .}

\section{Contribution of Ion Desolvation to Capacitance}

Figure 1 (c) depicts a representative nanopore embedded in microporous electrode. The nanopore is modelled as a structureless slit pore with pore width, and both surfaces are positively charged. When calculating the capacitance, the solvated ions are usually described with coarse-grained model. Here the restricted primitive model (RPM) is adopted, in which both the cation and anion are modelled as charged hard-sphere particles of the same size, and the solvent is treated as a dielectric continuum characterized with a fixed dielectric constant. The ionic size often takes the value of solvation diameter,.$^{\mathbf{2 0}, \mathbf{3 2}}$

Similar to the MDFT, the grand potential of a simple ionic system in the framework of CDFT can be formulated as a functional of local density distribution:

,$(5)$

where is the chemical potential of ionic species determined by the given bulk thermodynamic condition. is the non-electrostatic external potential, and in the coarse-grained model, unless otherwise specified elsewhere we adopt the hard-wall potential, i.e ., for and otherwise with being the perpendicular distance of the ionic particle within the pore to either nanoslit surface. is the intrinsic Helmholtz free energy, and it is generally composed of the ideal contribution and the excess one due to the intermolecular interaction :

. (6)

The ideal Helmholtz free energy of the system can be formulated exactly, reading:

,$(7)$

where, and is the thermal wavelength which is immaterial to the final calculation results. By minimizing the grand potential functional, i.e., , we can obtain the local density distribution of ionic particle at 
thermodynamic equilibrium:

, (8)

where is the excess part of . For the RPM model system, three contributions should be included in the excess Helmholtz free energy functional, including the excluded volume effect, the electrostatic correlation, and the direct Coulomb interaction ${ }^{50}$ The excluded volume effect is calculated by the modified fundamental measure theory (MFMT), ${ }^{\mathbf{5 1 - 5 2}}$ extended from the original FMT proposed by Rosenfeld. ${ }^{\mathbf{5 3}}$ By ignoring the higher-order correlation, the electrostatic correlation is derived from the second-order functional expansion of the excess Helmholtz free energy functional with respect to the bulk system. ${ }^{37}$ Following the mean-spherical approximation, ${ }^{\mathbf{5}}$ the excess Helmholtz free energy functional due to the contribution from direct Coulomb interaction can be formulated, and this contribution can be numerically solved by integrating the Poisson equation with the applied voltage, , on both slit surfaces. The detailed expressions of three contributions are given in the SI .

Because the structural and thermodynamic properties vary along the normal direction of nanoslit, we only consider the one-dimensional local density distribution of ionic particle, , in eq.(8). For a like-charged nanoslit, the surface charge density, , can be determined upon by neutralizing the net charge accumulation in the half-slit, i.e.,

,$(9)$

where is elementary charge, and is the valence carried on the ionic particle of $i$-th species. With the relation between the applied surface voltage and the resultant surface charge density, the integral capacitance of each like-charged nanoslit with pore width, hereafter designated as, can be calculated by .

The overall capacitance of a microporous electrode with the PSD can be calculated as:

, (10)

where the low limit in the integral is the smallest size of enterable pore, and it should be determined by the bare ion diameter, i.e.,. The upper limit, , should be the maximum pore size in the microporous electrode.

In experiment, the microporous electrode is often characterized with the average pore size, , in addition to the PSD. The averaged pore size is determined by: ${ }^{55}$

, (11)

Here, is the minimum measured pore size of nanopore in the electrode material. In this work, is directly extracted from the experimental data, which was determined through the argon adsorption isotherms. ${ }^{\mathbf{5 6}}$

\section{Results and Discussion}

\subsection{Solvation Diameter in Confined MeCN}

Both the radial distribution function and radial orientation distribution of $\mathrm{MeCN}$ surrounding $\mathrm{Na}^{+}$or $\mathrm{Cl}^{-}$in free space are calculated by using the MDFT in comparison with the relevant MD simulations. ${ }^{\mathbf{5}}$ The same set of system parameters is employed in the MDFT calculations: temperature $T=298 \mathrm{~K}$ and bulk MeCN number density $=1.139 \times 10^{-2}$ molecule $/ \AA^{3}$ (equivalently, the mass density, $0.777 \mathrm{~g} / \mathrm{cm}^{3}$ ), and the LJ parameters are presented in the SI .

Figure 2 (a) and (b) show the radial distribution functions of $\mathrm{MeCN}$, , surrounding $\mathrm{Na}^{+}$and $\mathrm{Cl}^{-}$, respectively. Here is the radial distance to the center of solute ion. The radial orientation distribution function of $\mathrm{MeCN}$ is calculated as:

,$(12)$

where is the orientation-dependent radial distribution function of $\mathrm{MeCN}$ solvent, and following our previous work $^{\mathbf{3 6}}$ it can be obtained straightforwardly from the local structure of MeCN solvent described with the orientation-dependent local density. The first peaks of radial distribution functions for $\mathrm{Na}^{+}$and $\mathrm{Cl}^{-}$are 
remarkably larger than the other peaks, indicating that the solvation radii of ions are closely dependent on the MeCN solvent within the first solvation shell.

Figure 2 (c) and (d) plot the radial orientation distribution functions of $\mathrm{MeCN}$ surrounding $\mathrm{Na}^{+}$and $\mathrm{Cl}^{-}$, respectively. The radial orientation distribution functions display an oscillatory feature, showing that the radial density distribution is sensitive to the orientation angle. Namely, at different orientation angles, the radial density distributions can be very different. Indeed, owing to the strong electrostatic interaction between the solute ion and MeCN solvent, the nitrogen site of MeCN orients preferentially towards $\mathrm{Na}^{+}$ within the first solvation shell. Opposite situation can be found with the solute of $\mathrm{Cl}^{-}$. Although there is a small deviation between the MDFT predictions and MD simulation results, the density distributions within the first solvation shell are in excellent agreements. This shows that the MDFT provides an accurate and feasible method to calculate the local density and orientation angle of MeCN surrounding solute anion or cation.

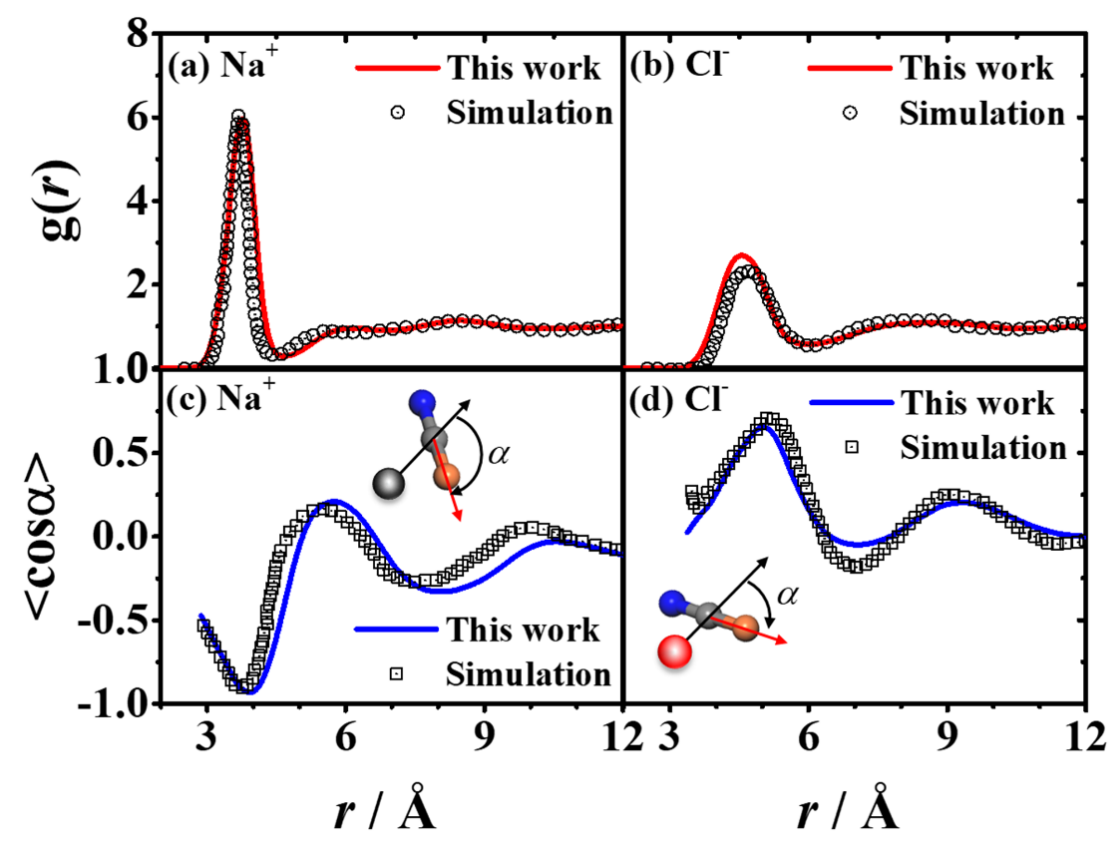

Figure 2 . ( $\mathrm{a}, \mathrm{b})$ The radial distribution functions and (c, d) the radial orientation distribution functions of MeCN predicted by the MDFT in comparison with relevant MD simulation. ${ }^{57} \mathrm{Na}^{+}$: left panel; $\mathrm{Cl}^{-}$: right panel.

As the radial distribution function is highly orientation-dependent, the structural criterion involving both local density and orientation angle are utilized to determine the solvation diameters of ions in MeCN. The essential idea is that at certain orientations the local densities within the first solvation shell are very low and make no contribution to the solvation number. To justify this postulation, we compute the molecular number within the solvent shell at each orientation angle:

where is the molecular number at the specific orientation within the solvent shell determined by the first density valley position, as discussed in the eq.(2).

The reduced number, , for $\mathrm{Cl}^{-}$are plotted inFigure 3 (a). Interestingly, most of MeCN molecules within the first shell surrounding $\mathrm{Cl}^{-}$distribute with the orientation ranging from 0.5 to 1 , and the maximum reduced number is found at $=0.75$. This trend confirms the above analysis. To distinguish the non-contributing 
molecules, we introduce a critical value of, below which the corresponding reduced number is small and thus makes no contribution to the solvation diameter. This critical value should be sort of universal, and valid for many other simple ions as well. To determine this critical value, we take the measured solvation diameter of $\mathrm{Cl}^{-}$from experiment (i.e., $7.52 \AA^{\mathbf{5 8}}$ ) as a benchmark, and then determine critical structural orientation as $=$ 0.68 . The applicability of this criterion is tested for evaluating the solvation diameter of other simple anions in MeCN.Figure 3 (b) shows the solvation diameters of $\mathrm{Cl}^{-}, \mathrm{Br}^{-}$and $\mathrm{I}^{-}$in $\mathrm{MeCN}$ predicted by the MDFT. As can be seen, the predictions of $\mathrm{Br}^{-}$and $\mathrm{I}^{-}$are in a good agreement with the experimental measurements. Therefore, we speculate that this structural criterion is applicable for predicting the solvation diameters of anions in confined MeCN solvent.
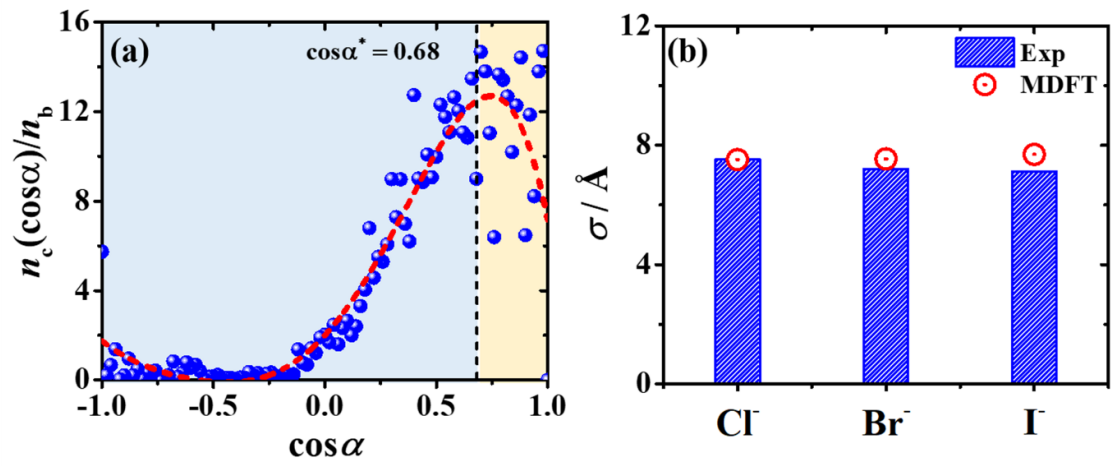

Figure 3 . (a) Reduced MeCN number as a function of for $\mathrm{Cl}^{-}$. (b) Predicted solvation diameters for anions in comparison with experimental results. ${ }^{58}$ The red dash line in the right figure is drawn to guide the eye.

To further examine the accuracy of MDFT for predicting the MeCN structure in confined space, the local density distribution of $\mathrm{MeCN}$ in a nanoslit is calculated and then compared with simulation results. In the simulation, the nanoslit is constructed with two parallel graphene monolayers with separation $=42 \AA$. The simulation are performed with standard molecular dynamics simulation package, in which the graphene is described with full-atomic model, and the three-site model ${ }^{\mathbf{4 2}}$ for the MeCN liquid at $298 \mathrm{~K}$ is utilized. The simulation details are provided in the SI . The same model system is adopted in the MDFT calculation. Technically, we place the left graphene monolayer at $=0.0$ while the right one at .Figure 4 (a) plots the predicted normalized density distributions of MeCN, , along the direction from both the MDFT and simulation. The normalized density distribution from the MDFT is computed by taking average of the angular-dependent local density over all MeCN orientations. The comparison shows that the MDFT prediction well captures the layer-by-layer structure of MeCN near the wall, in excellent consistency with the simulation result. For the sake of clear illustration, the corresponding two-dimensional density map near the wall is depicted inFigure $4(\mathrm{~b})$.

Next, we analyze the solvation diameter of ion in confined MeCN. For reducing the computational cost on the external potential, we reconstruct the nanoslit with two flat and structureless graphene-like layers, in which the interaction between the $\mathrm{MeCN}$ solvent and neutral flat wall is described by the 10-4 potential. ${ }^{49}$ The solute ion is placed at the center of the nanoslit and immersed in the confined MeCN at ambient condition. Taking $\mathrm{Cl}^{-}$as a case study, we compute the solvation number and solvation diameter of $\mathrm{Cl}^{-}$in different nanoslits by using the MDFT, as shown in Figure 4 (c) and (d)respectively. In the nanoslit with sufficiently large pore width, the solvation number and the solvation diameter recover to their corresponding bulk values. As the pore width decreases, the solvation number and the solvation diameter first oscillate around their bulk values, and then monotonically decrease until reaching the limits. This oscillative decrement of the solvation diameter unravels the nature of ion desolvation in confined solvent, and it's essentially consistent with the reported variation trend of coordination number in confined systems. For example, Shao et al., ${ }^{45}$ reported a non-monotonic variation of the coordination number of $\mathrm{Na}^{+}$and $\mathrm{K}^{+}$in confined water in nanotube as 
decreasing the nanotube size.
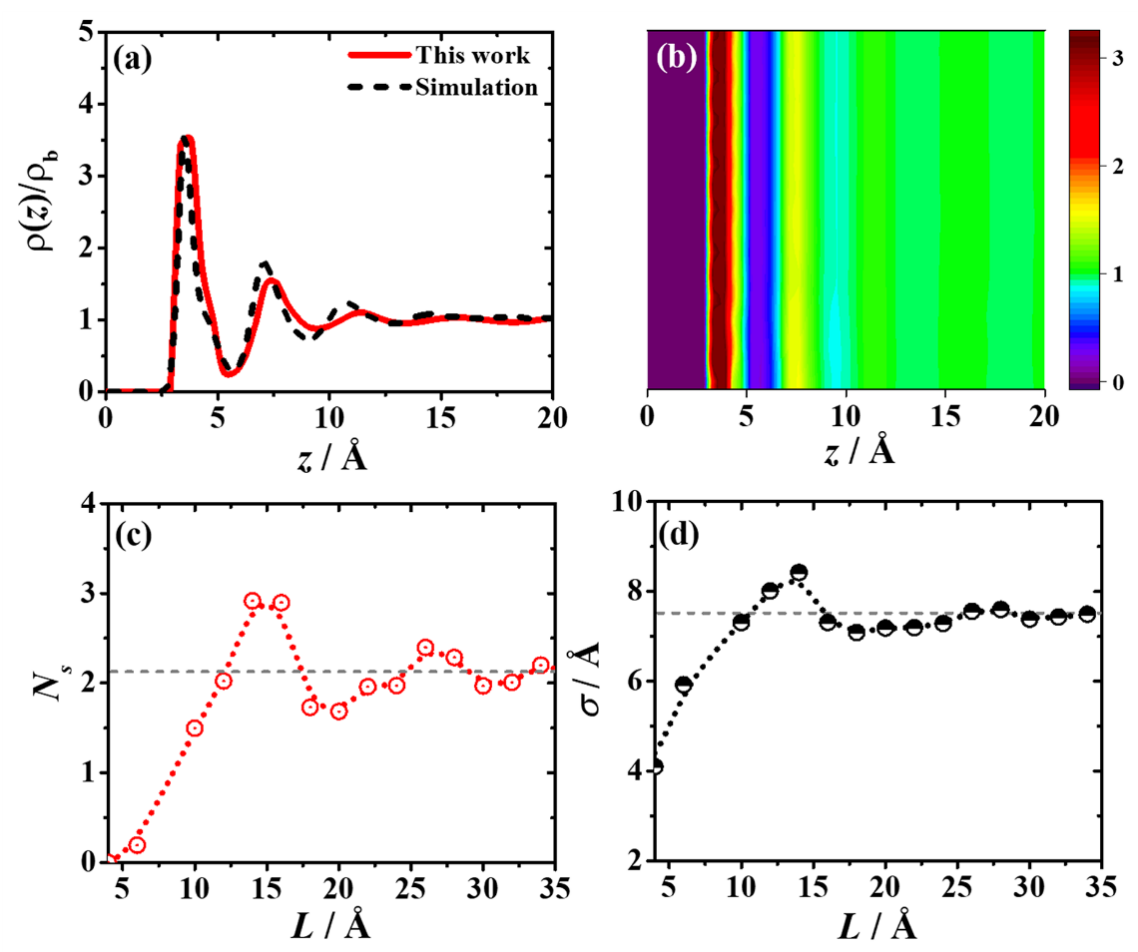

Figure 4 . (a) Normalized density distribution of $\mathrm{MeCN}$ near a full-atomic graphene wall, and (b) the corresponding layer-by-layer two-dimensional density map. (c) The solvation number and (d) solvation diameter of $\mathrm{Cl}^{-}$versus the pore width of nanoslit. The grey dash lines represent the corresponding bulk values. The red and black dotted lines are drawn to guide the eye.

\subsection{Microstructure of Liquid Electrolytes in Nanoslit}

The electrochemical properties of supercapacitors are determined by the microstructure of electrolytes in each nanopore. The electrolytes are usually modeled with RPM model with the ion size identical with its solvation diameter. ${ }^{\mathbf{2 0}, \mathbf{3 2}}$ To explore the ion size effect, the density distributions of cation and anion are investigated in the same nanopore with identical ion concentration. Three typical sizes of cation and anion () are chosen, i.e ., $=0.33 \mathrm{~nm}, 0.57 \mathrm{~nm}$, and $0.73 \mathrm{~nm}$, which corresponds to, respectively, the bare ion size, the solvation diameter in confined $\mathrm{MeCN}$ with pore size $=0.92 \mathrm{~nm}$, and the solvation diameter in bulk MeCN. The other system parameters are given as follows: the relative dielectric constant $=1.0$, the system temperature $=298 \mathrm{~K}$, the surface voltage $=1.15 \mathrm{~V}$, and the bulk ion concentration $=1.50 \mathrm{M}$. The predicted ion density profiles by the CDFT are shown in Figure 5 . As expected, anions accumulate near the positively charged surfaces while cations are repelled to the center of nanoslit. In addition, we note that the ion distribution is sensitive to the solvation diameter. Specifically, when increasing the solvation diameter of ion, the contact value of anion increases, and the accumulation of both cations and anions are enhanced. 

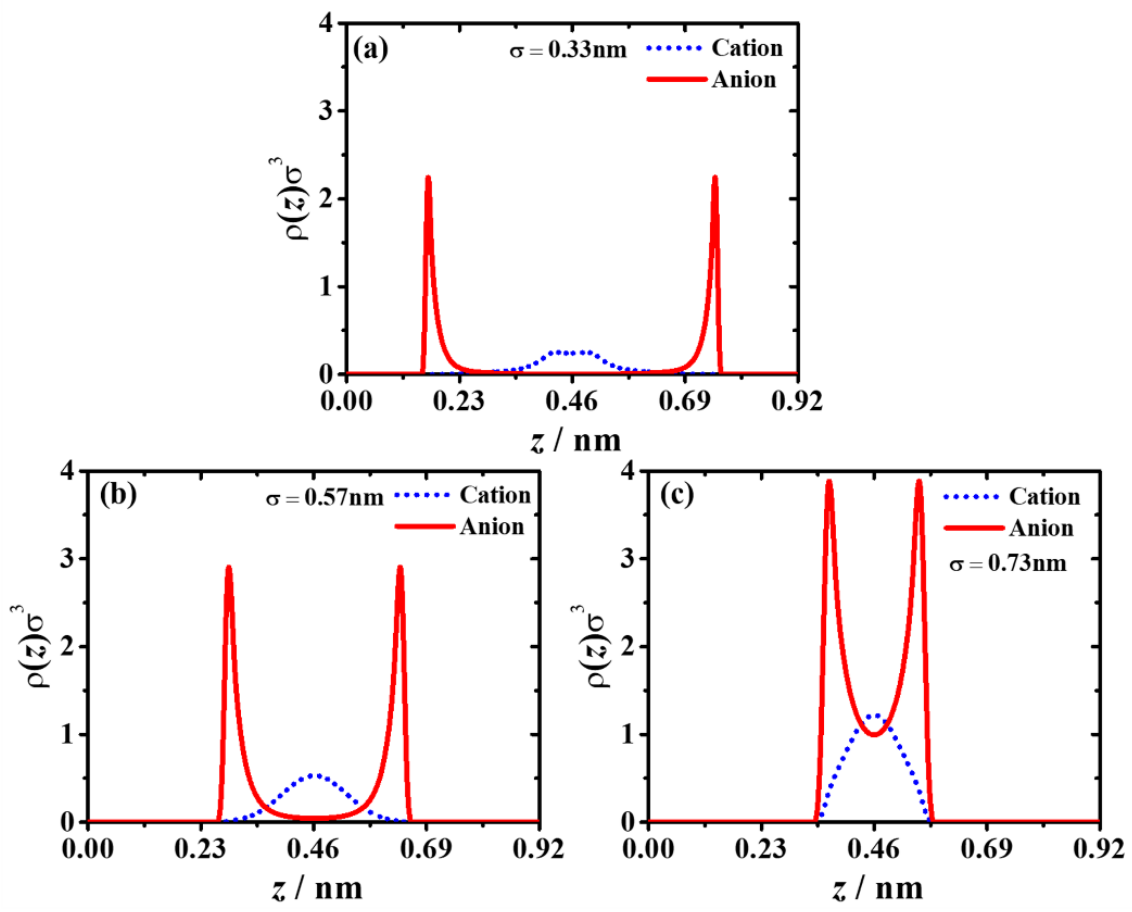

Figure 5. Density distributions of cation and anion within the nanoslit with pore size $=0.92 \mathrm{~nm}$. Three ionic diameters: $(\mathrm{a})=0.33 \mathrm{~nm},(\mathrm{~b})=0.57 \mathrm{~nm}$ and $(\mathrm{c})=0.73 \mathrm{~nm}$ are considered, corresponding to the bare ion size, the solvation diameter in confined MeCN, and the solvation diameter in bulk MeCN.

\subsection{Contribution of Ion Desolvation to Capacitance}

Now we start to analyze the ion desolvation effect on capacitance. To validate the feasibility of CDFT in combination with the RPM coarse-grained model for predicting capacitance, comparisons between the CDFT calculation and relevant simulation ${ }^{59}$ are performed and shown in Figure $\mathbf{6}$. In the simulation, the electrochemical properties of hard-sphere ions near a planar hard wall are investigated, in which the relative dielectric constant is set as $=78.50$ for mimicking aqueous solution, and a moderate electrolyte concentration is adopted as $=1.0 \mathrm{M}$ with equal diameters of cation and anion $=0.40 \mathrm{~nm}$. The system temperature is $=$ 298.15 K. The identical system parameters are used in the CDFT calculation except that a nanoslit with pore width is applied. The comparison shows that our coarse-grained-model based CDFT calculation gives satisfactory predictions on the relation between surface voltage and surface charge density, and the relation between the capacitance and the surface charge density except a little overestimate at the peaks.
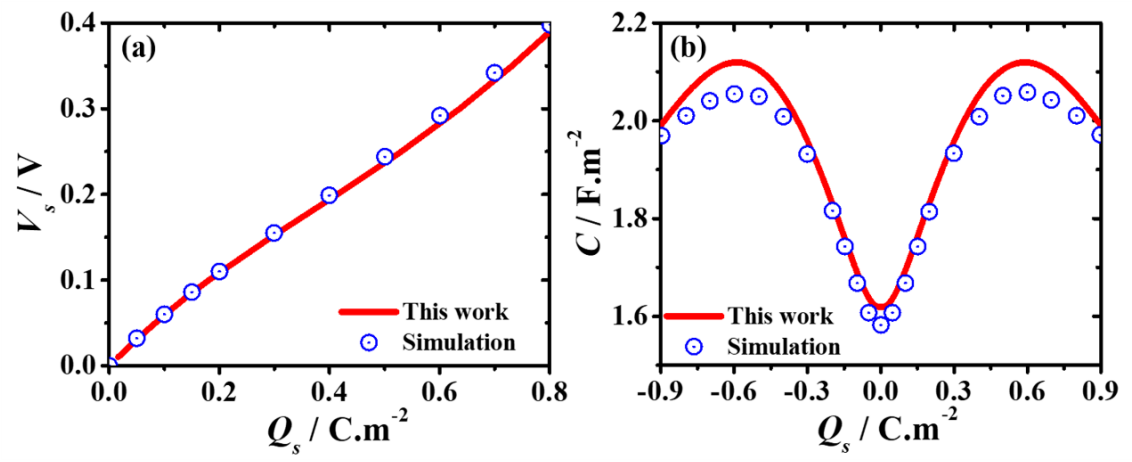
Figure 6 . (a) Surface voltage as a function of surface charge density; and (b) the capacitance of nanoslit versus the surface charge density, predicted through the CDFT in comparison with the simulation reported by Gorniak et al. ${ }^{59}$

By using the MDFT in combination with the critical structural orientation $=0.68$, we first calculate the solvation diameters of ions in confined $\mathrm{MeCN}$ within the nanoslits with different pore widths. Here, the anion of $\mathrm{BF}_{4}{ }^{-}$is considered which is modeled as a LJ particle carrying one elementary charge. The LJ parameters are presented in the SI with the size of bare ion $=0.33 \mathrm{~nm}$.Figure 7 (a) shows the dependence of the predicted solvation diameter versus the pore width of nanoslit, i.e ., . The solvation diameter varies along the pore width, displaying an oscillative decline when reducing the pore width.

By incorporating the ion solvation diameter into the solvation-diameter-dependent coarse-grained model, and assuming that the cation and anion have equal size, the capacitances of the nanoslits involving MeCN-based electrolyte with different pore widths are calculated through CDFT, as shown in Figure 7 (b) . Here, the bulk ion concentration $=1.50 \mathrm{M}$ and the other system parameters are the same as those in Figure 5 . For comparison, the capacitances without considering ion desolvation are calculated as well, in which all parameters are the same except that a uniform ion size is adopted as $=0.73 \mathrm{~nm}$ corresponding to the solvation diameter of $\mathrm{BF}_{4}^{-}$in bulk MeCN. Conventionally, the ion solvation diameter in bulk solution has been employed in theoretical calculation. ${ }^{60}$
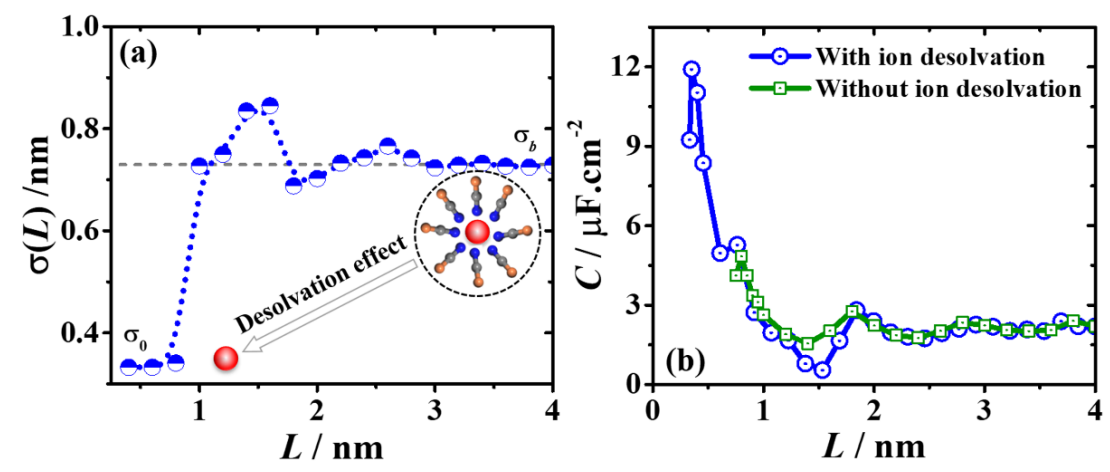

Figure 7 . (a) Predicted solvation diameter of $\mathrm{BF}_{4}{ }^{-}$varying with the pore width of nanoslit. (b) Capacitance with and without ion desolvation effect varying with the pore size of nanoslit. The blue dotted line is drawn to guide the eye.

Overall, the predicted capacitance in terms of pore width displays an oscillating feature, and besides, the capacitances in both cases become almost identical as the pore width exceeds $2.0 \mathrm{~nm}$. This is because the solvation diameter of $\mathrm{BF}_{4}^{-}$gradually recovers to its bulk value when increasing the pore width. Nevertheless, two important different features can be found between the capacitances considering ion-desolvation effect and that without ion-desolvation effect. Firstly, owing to the pore size limit, the capacitance with uniform ion size vanishes when the pore width is less than $0.73 \mathrm{~nm}$; while for the case considering ion-desolvation, as the ion size can decrease down to $0.33 \mathrm{~nm}$, the capacitance sharply increases when the pore width decreases from 0.73 to $0.33 \mathrm{~nm}$, generally according with the experimental observation. ${ }^{\mathbf{1 2}}$ Secondly, an obvious deviation in the capacitance can be observed when the pore width varies from $1.0 \mathrm{~nm}$ to $2.0 \mathrm{~nm}$. This is likely due to the mismatch between and resulting from the oscillation of solvation diameter in confined MeCN.

The overall capacitance, , is calculated with the help of eq.(10) and the PSD extracted from the measurement of TiC-CDC microporous material. ${ }^{56}$ Two types of overall capacitances are calculated, i.e ., the ones with and without ion desolvation. The calculated overall capacitances versus the average pore size are plotted in Figure $\mathbf{8}$ in comparison with the experimental measurement from Chmiola et al. ${ }^{12}$ The predicted overall capacitance involving ion desolvation effect nicely agrees well with the experimental observation in both the magnitude and the variation trend along with the average pore size. Particularly, the sharp increase of 
capacitance is well captured as decreasing the average pore size. However, the overall capacitances without ion desolvation are overall much smaller than the experimental values, and in addition, the sharp increase of capacitance in nanopore is not captured. These differences highlight the importance of ion-desolvation effect in determining the capacitance of microporous electrode. Furthermore, the agreement between our predictions and experimental measurements shows that the capacitances of practical microporous electrodes involving liquid electrolytes can be quantitatively predicted by the proposed multiscale molecular approach.

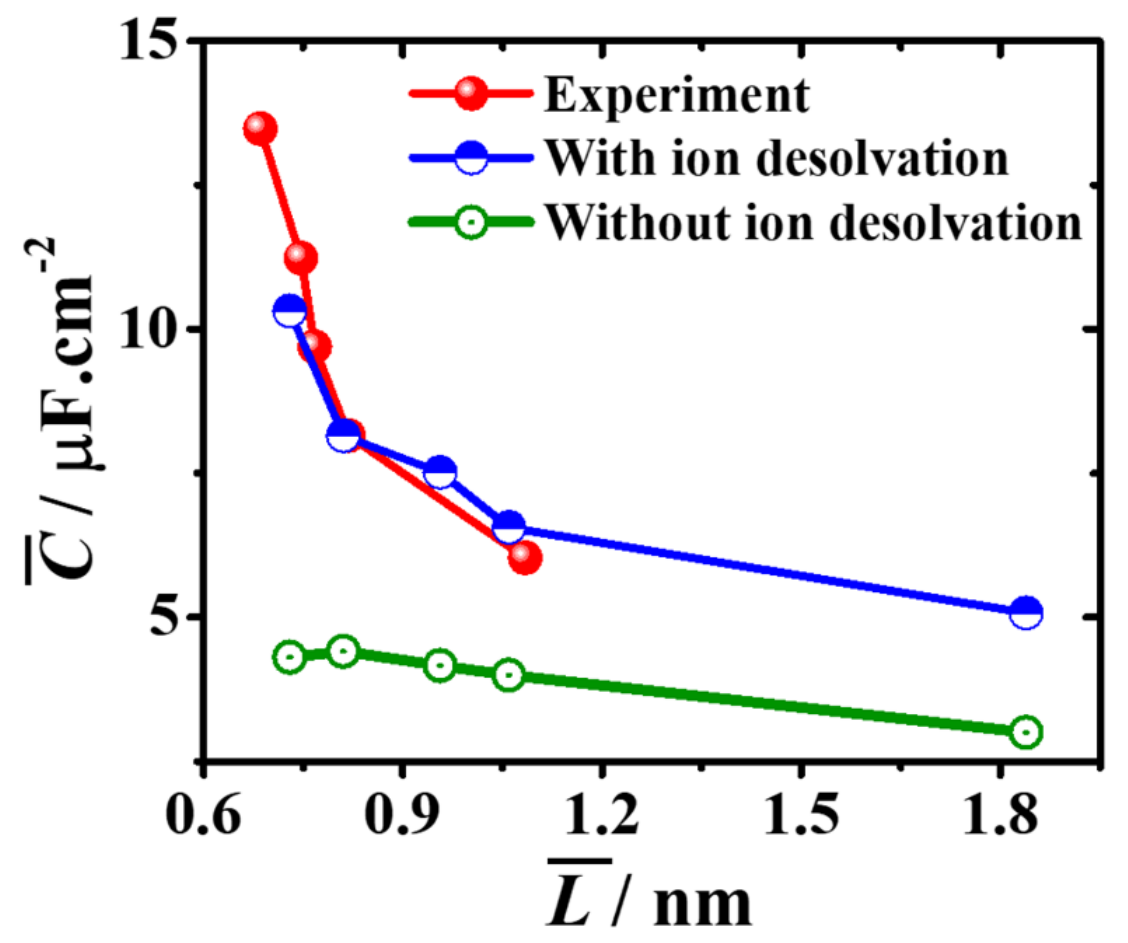

Figure 8 . Overall capacitances of the microporous electrode with and without the ion desolvation versus the average pore size. The experimental capacitances are from the experiment reported by Chmiola et al. ${ }^{\mathbf{1 2}}$

\section{Conclusion}

The contribution of ion desolvation to the capacitance of microporous electrodes is investigated by combining the MDFT and CDFT. With the help of MDFT, we first develop a criterion to determine the solvation diameter of ion in $\mathrm{MeCN}$, and then evaluate the ion solvation diameters in the MeCN confined in nanopores with different pore sizes at ambient condition. Particularly, the relation between the solvation diameter and pore size is unraveled. Interestingly, we find that the solvation diameter of ion displays an oscillative decline to the bare ion size when decreasing the pore size.

With the help of this pore-size-dependent solvation diameter, we are able to incorporate the coarse-grained model of solvated ion with the PSD of practical microporous electrode extracted from experimental measurement, and quantitatively predict the capacitance by means of CDFT. This work not only provides a multiscale molecular approach to evaluate the contribution of ion desolvation to electrochemical properties of microporous electrodes, but also casts insights into the design and optimization of supercapacitors.

\section{Acknowledgements}

This work is supported by National Natural Science Foundation of China (Nos. 91934302, U1707602, and 21878078), and the 111 Project of China (No. B08021). L.Q. is grateful to the China Scholarship Council. 
We thank Dr. Jiabo Tao for providing the simulation data of MeCN in slit pores for our comparison.

\section{References}

1. Lu, M., Supercapacitors: materials, systems, and applications . John Wiley \& Sons: 2013.

2. Simon, P.; Gogotsi, Y., Materials for electrochemical capacitors. Nature Materials 2008, 7 (11), 845-854.

3. Merlet, C.; Rotenberg, B.; Madden, P. A.; Taberna, P.-L.; Simon, P.; Gogotsi, Y.; Salanne, M., On the molecular origin of supercapacitance in nanoporous carbon electrodes. Nature Materials 2012,11 (4), 306-310.

4. Zhan, C.; Lian, C.; Zhang, Y.; Thompson, M. W.; Xie, Y.; Wu, J.; Kent, P. R.; Cummings, P. T.; Jiang, D. e.; Wesolowski, D. J., Computational insights into materials and interfaces for capacitive energy storage. Advanced Science 2017, \& (7), 1700059.

5. Vatamanu, J.; Bedrov, D., Capacitive Energy Storage: Current and Future Challenges. Journal of Physical Chemistry Letters2015, 6 (18), 3594-3609.

6. Simon, P.; Gogotsi, Y., Charge storage mechanism in nanoporous carbons and its consequence for electrical double layer capacitors. Philosophical Transactions Mathematical Physical E Engineering Sciences 2010, 368 (1923), 3457-3467.

7. Salanne, M.; Rotenberg, B.; Naoi, K.; Kaneko, K.; Taberna, P.-L.; Grey, C. P.; Dunn, B.; Simon, P., Efficient storage mechanisms for building better supercapacitors. Nature Energy 2016,1 (6), 1-10.

8. Huang, S.; Zhu, X.; Sarkar, S.; Zhao, Y., Challenges and opportunities for supercapacitors. APL Materials 2019,7 (10), 100901.

9. Simon, P.; Gogotsi, Y.; Dunn, B., Where do batteries end and supercapacitors begin? Science 2014, 343 (6176), 1210-1211.

10. Gogotsi, Y.; Nikitin, A.; Ye, H.; Zhou, W.; Fischer, J. E.; Yi, B.; Foley, H. C.; Barsoum, M. W., Nanoporous carbide-derived carbon with tunable pore size. Nature materials 2003, 2 (9), 591-594.

11. Dash, R.; Chmiola, J.; Yushin, G.; Gogotsi, Y.; Laudisio, G.; Singer, J.; Fischer, J.; Kucheyev, S., Titanium carbide derived nanoporous carbon for energy-related applications. Carbon 2006, 44 (12), 24892497.

12. Chmiola, J.; Yushin, G.; Gogotsi, Y.; Portet, C.; Simon, P.; Taberna, P.-L., Anomalous increase in carbon capacitance at pore sizes less than 1 nanometer. Science 2006, 313 (5794), 1760-1763.

13. Largeot, C.; Portet, C.; Chmiola, J.; Taberna, P.-L.; Gogotsi, Y.; Simon, P., Relation between the ion size and pore size for an electric double-layer capacitor. Journal of the American Chemical Society2008, 130 (9), 2730-2731.

14. Kondrat, S.; Georgi, N.; Fedorov, M. V.; Kornyshev, A. A., A superionic state in nano-porous doublelayer capacitors: insights from Monte Carlo simulations. Physical Chemistry Chemical Physics2011, 13 (23), 11359-11366.

15. Shim, Y.; Kim, H. J., Nanoporous Carbon Supercapacitors in an Ionic Liquid: A Computer Simulation Study. Acs Nano 2010,4 (4), 2345-2355.

16. Feng, G.; Cummings, P. T., Supercapacitor Capacitance Exhibits Oscillatory Behavior as a Function of Nanopore Size. Journal of Physical Chemistry Letters 2011, 2 (22), 2859-2864.

17. Wu, P.; Huang, J.; Meunier, V.; Sumpter, B. G.; Qiao, R., Complex capacitance scaling in ionic liquids-filled nanopores. Acs Nano2011, 5 (11), 9044-9051. 
18. Xing, L.; Vatamanu, J.; Borodin, O.; Bedrov, D., On the Atomistic Nature of Capacitance Enhancement Generated by Ionic Liquid Electrolyte Confined in Subnanometer Pores. Journal of Physical Chemistry Letters 2013, 4 (1), 132-140.

19. Huang, J.; Sumpter, B. G.; Meunier, V., Theoretical model for nanoporous carbon supercapacitors. Angewandte Chemie2008, 120 (3), 530-534.

20. Jiang, D.-e.; Jin, Z.; Wu, J., Oscillation of capacitance inside nanopores. Nano Letters 2011, 11 (12), 5373-5377.

21. Prehal, C.; Koczwara, C.; Jäckel, N.; Schreiber, A.; Burian, M.; Amenitsch, H.; Hartmann, M. A.; Presser, V.; Paris, O., Quantification of ion confinement and desolvation in nanoporous carbon supercapacitors with modelling and in situ X-ray scattering. Nature Energy2017, 2 (3), 1-8.

22. Xu, K.; Shao, H.; Lin, Z.; Merlet, C.; Feng, G.; Zhu, J.; Simon, P., Computational Insights into Charge Storage Mechanisms of Supercapacitors. Energy 83 Environmental Materials 2020,3 (3), 235-246.

23. Qing, L.; Li, Y.; Tang, W.; Zhang, D.; Han, Y.; Zhao, S., Dynamic Adsorption of Ions into Like-Charged Nanospace: A Dynamic Density Functional Theory Study. Langmuir 2019, 35 (12), 4254-4262.

24. Lian, C.; Jiang, D.-e.; Liu, H.; Wu, J., A generic model for electric double layers in porous electrodes. The Journal of Physical Chemistry C 2016, 120 (16), 8704-8710.

25. Feng, G.; Jiang, D.-e.; Cummings, P. T., Curvature effect on the capacitance of electric double layers at ionic liquid/onion-like carbon interfaces. Journal of Chemical Theory and Computation 2012, 8 (3), 10581063.

26. Jackel, N.; Simon, P.; Gogotsi, Y.; Presser, V., Increase in capacitance by subnanometer pores in carbon. ACS Energy Letters 2016, 1 (6), 1262-1265.

27. Chmiola, J.; Largeot, C.; Taberna, P. L.; Simon, P.; Gogotsi, Y., Desolvation of ions in subnanometer pores and its effect on capacitance and double-layer theory. Angewandte Chemie International Edition 2008, $47(18), 3392-3395$.

28. Urita, K.; Urita, C.; Fujita, K.; Horio, K.; Yoshida, M.; Moriguchi, I., The ideal porous structure of EDLC carbon electrodes with extremely high capacitance. Nanoscale 2017, 9 (40), 15643-15649.

29. Borchardt, L.; Leistenschneider, D.; Haase, J.; Dvoyashkin, M., Revising the concept of pore hierarchy for ionic transport in carbon materials for supercapacitors. Advanced Energy Materials 2018, 8 (24), 1800892.

30. Morales, C. M.; Thompson, W. H., Simulations of Infrared Spectra of Nanoconfined Liquids: Acetonitrile Confined in Nanoscale, Hydrophilic Silica Pores. The Journal of Physical Chemistry A 2009,113 (10), 19221933.

31. Srimuk, P.; Lee, J.; Budak, O. z.; Choi, J.; Chen, M.; Feng, G.; Prehal, C.; Presser, V., In situ tracking of partial sodium desolvation of materials with capacitive, pseudocapacitive, and battery-like charge/discharge behavior in aqueous electrolytes. Langmuir 2018, 34 (44), 13132-13143.

32. Jiang, J.; Cao, D.; Jiang, D.-e.; Wu, J., Kinetic charging inversion in ionic liquid electric double layers. The Journal of Physical Chemistry Letters 2014, 5 (13), 2195-2200.

33. Merlet, C.; Pean, C.; Rotenberg, B.; Madden, P. A.; Daffos, B.; Taberna, P.-L.; Simon, P.; Salanne, M., Highly confined ions store charge more efficiently in supercapacitors. Nature Communications 2013, 4 (1), 1-6.

34. Pean, C.; Daffos, B.; Rotenberg, B.; Levitz, P.; Haefele, M.; Taberna, P.-L.; Simon, P.; Salanne, M., Confinement, desolvation, and electrosorption effects on the diffusion of ions in nanoporous carbon electrodes. Journal of the American Chemical Society2015, 137 (39), 12627-12632. 
35. Thompson, W. H., Solvation dynamics and proton transfer in nanoconfined liquids. Annual Review of Physical Chemistry 2011, 62 , 599-619.

36. Qing, L.; Tao, J.; Yu, H.; Jiang, P.; Qiao, C.; Zhao, S.; Liu, H., A molecular model for ion dehydration in confined water. AIChE Journal 2020, 66 (6), e16938.

37. Zhao, S.; Liu, Y.; Chen, X.; Lu, Y.; Liu, H.; Hu, Y., Unified framework of multiscale density functional theories and its recent applications. In Advances in Chemical Engineering, Elsevier: 2015; Vol. 47, pp 1-83.

38. Lian, C.; Liu, H., Classical Density Functional Theory Insights for Supercapacitors. In SupercapacitorsTheoretical and Practical Solutions, 2018.

39. Qing, L.; Lei, J.; Zhao, T.; Qiu, G.; Ma, M.; Xu, Z.; Zhao, S., Effects of Kinetic Dielectric Decrement on Ion Diffusion and Capacitance in Electrochemical Systems. Langmuir 2020, 36(15), 4055-4064.

40. Jiang, D.-e.; Meng, D.; Wu, J., Density functional theory for differential capacitance of planar electric double layers in ionic liquids. Chemical Physics Letters 2011, 504(4-6), 153-158.

41. Gouaux, E.; MacKinnon, R., Principles of selective ion transport in channels and pumps. Science 2005, 310 (5753), 1461-1465.

42. Edwards, D. M.; Madden, P. A.; McDonald, I. R., A computer simulation study of the dielectric properties of a model of methyl cyanide: I. The rigid dipole case. Molecular Physics1984, 51 (5), 11411161.

43. Liu, Y.; Zhao, S.; Wu, J., A site density functional theory for water: Application to solvation of amino acid side chains. Journal of Chemical Theory and Computation 2013, 9 (4), 1896-1908.

44. Zhou, J.; Lu, X.; Wang, Y.; Shi, J., Molecular dynamics study on ionic hydration. Fluid Phase Equilibria 2002,194, 257-270.

45. Shao, Q.; Zhou, J.; Lu, L.; Lu, X.; Zhu, Y.; Jiang, S., Anomalous hydration shell order of Na+ and K+ inside carbon nanotubes. Nano Letters 2009, 9 (3), 989-994.

46. Barker, J. A.; Henderson, D., Perturbation theory and equation of state for fluids. II. A successful theory of liquids. The Journal of Chemical Physics 1967, 47 (11), 4714-4721.

47. Zhao, S.; Jin, Z.; Wu, J., New Theoretical Method for Rapid Prediction of Solvation Free Energy in Water. The Journal of Physical Chemistry B 2011, 115 (21), 6971-6975.

48. Zhao, S.; Ramirez, R.; Vuilleumier, R.; Borgis, D., Molecular density functional theory of solvation: From polar solvents to water. The Journal of Chemical Physics 2011, 134 (19), 194102.

49. Siderius, D. W.; Gelb, L. D., Extension of the Steele 10-4-3 potential for adsorption calculations in cylindrical, spherical, and other pore geometries. The Journal of Chemical Physics2011, 135 (8), 084703.

50. Wu, J.; Li, Z., Density-functional theory for complex fluids. Annual Review of Physical Chemistry 2007, $58,85-112$.

51. Yu, Y. X.; Wu, J., Structures of hard-sphere fluids from a modified fundamental-measure theory. Journal of Chemical Physics2002, 117 (22), 10156-10164.

52. Roth, R.; Evans, R.; Lang, A.; Kahl, G., Fundamental measure theory for hard-sphere mixtures revisited: the White Bear version.Journal of Physics: Condensed Matter 2002, 14 (46), 12063.

53. Rosenfeld, Y., Structure and effective interactions in multi-component hard-sphere liquids: the fundamental-measure density functional approach. Journal of Physics: Condensed Matter 2002, 14 (40), 9141-9152.

54. Blum, L.; Rosenfeld, Y., Relation between the free energy and the direct correlation function in the mean spherical approximation. Journal of Statistical Physics 1991, 63 (5-6), 1177-1190. 
55. Hansen, J.-P.; McDonald, I. R., Theory of simple liquids . Elsevier: 1990.

56. Laudisio, G.; Dash, R. K.; Singer, J. P.; Yushin, G.; Gogotsi, Y.; Fischer, J. E., Carbide-derived carbons: a comparative study of porosity based on small-angle scattering and adsorption isotherms.Langmuir 2006, 22 (21), 8945-8950.

57. Guardia, E.; Pinzon, R., On the solvation shell of $\mathrm{Na}^{+}$and $\mathrm{Cl}^{-}$ions in acetonitrile: A computer simulation study. Journal of Molecular Liquids 2000, 85 (1-2), 33-44.

58. Della Monica, M.; Senatore, L., Solvated radius of ions in nonaqueous solvents. The Journal of Physical Chemistry1970, 74 (1), 205-207.

59. Gorniak, R.; Lamperski, S., Investigation of the electrical double layer with a graphene electrode by the grand canonical Monte Carlo simulation. The Journal of Physical Chemistry C 2014,118 (6), 3156-3161.

60. Kondrat, S.; Perez, C.; Presser, V.; Gogotsi, Y.; Kornyshev, A., Effect of pore size and its dispersity on the energy storage in nanoporous supercapacitors. Energy \& Environmental Science 2012, 5 (4), 6474-6479.

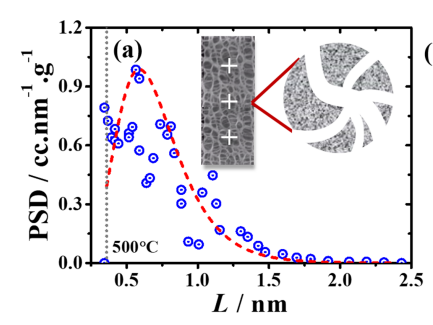

Microporous TiC-CDC

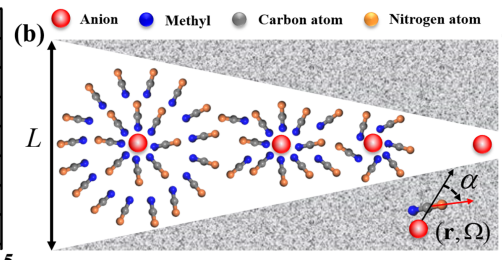

Ion Desolvation

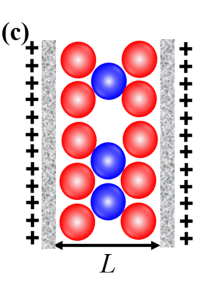

Individual Nanoslit

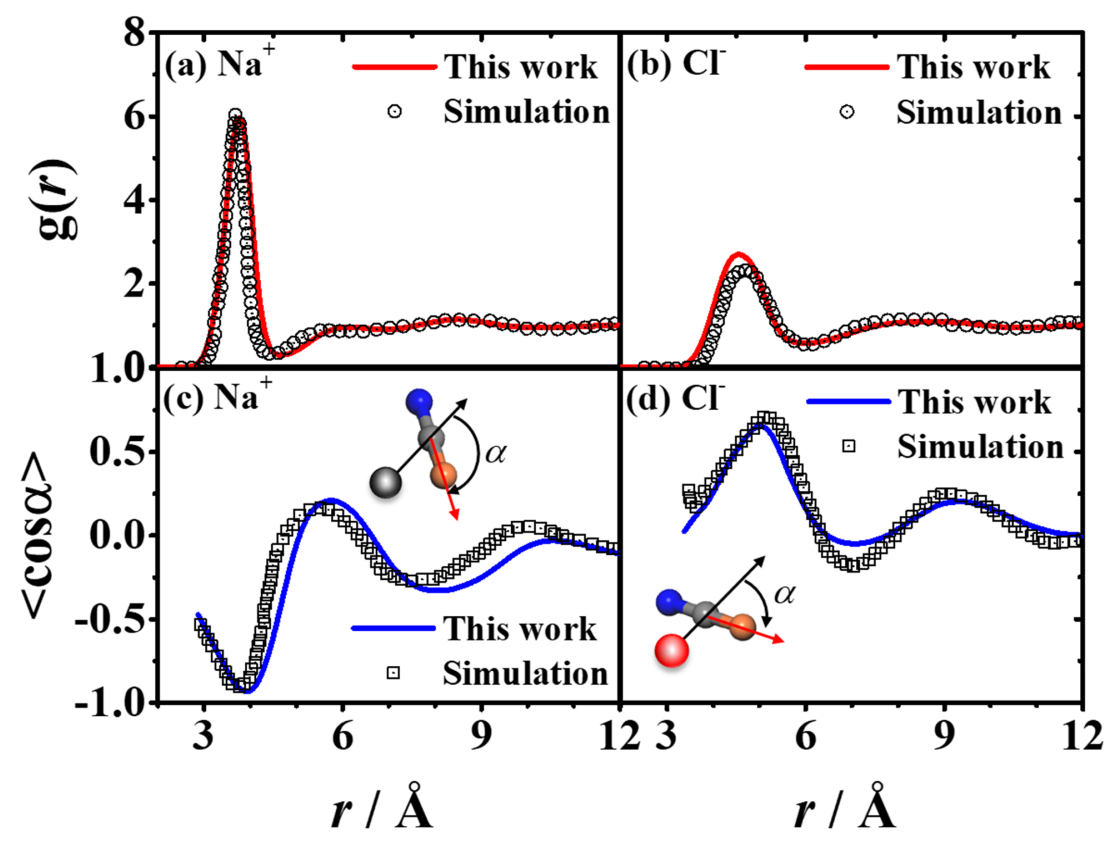



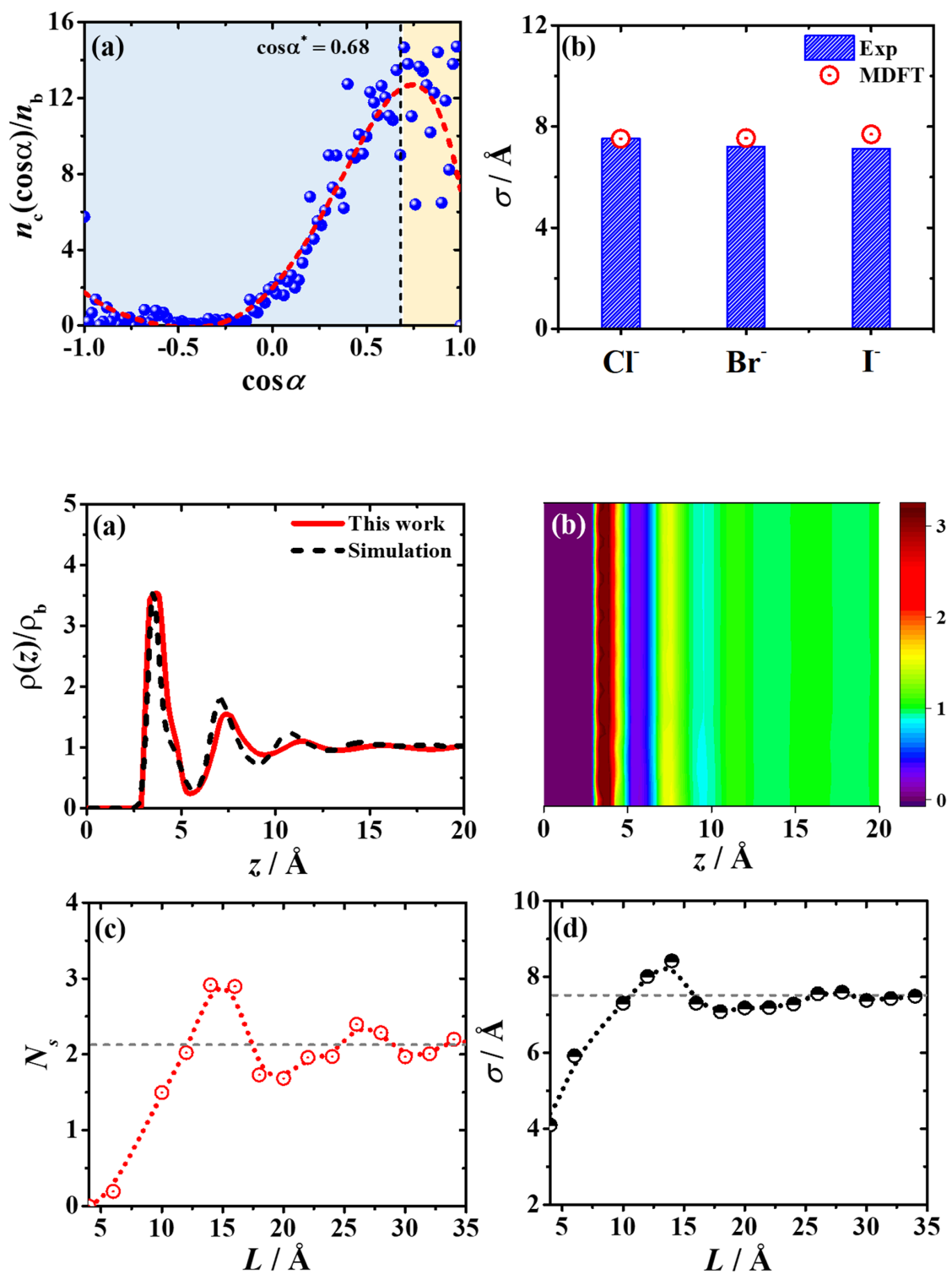

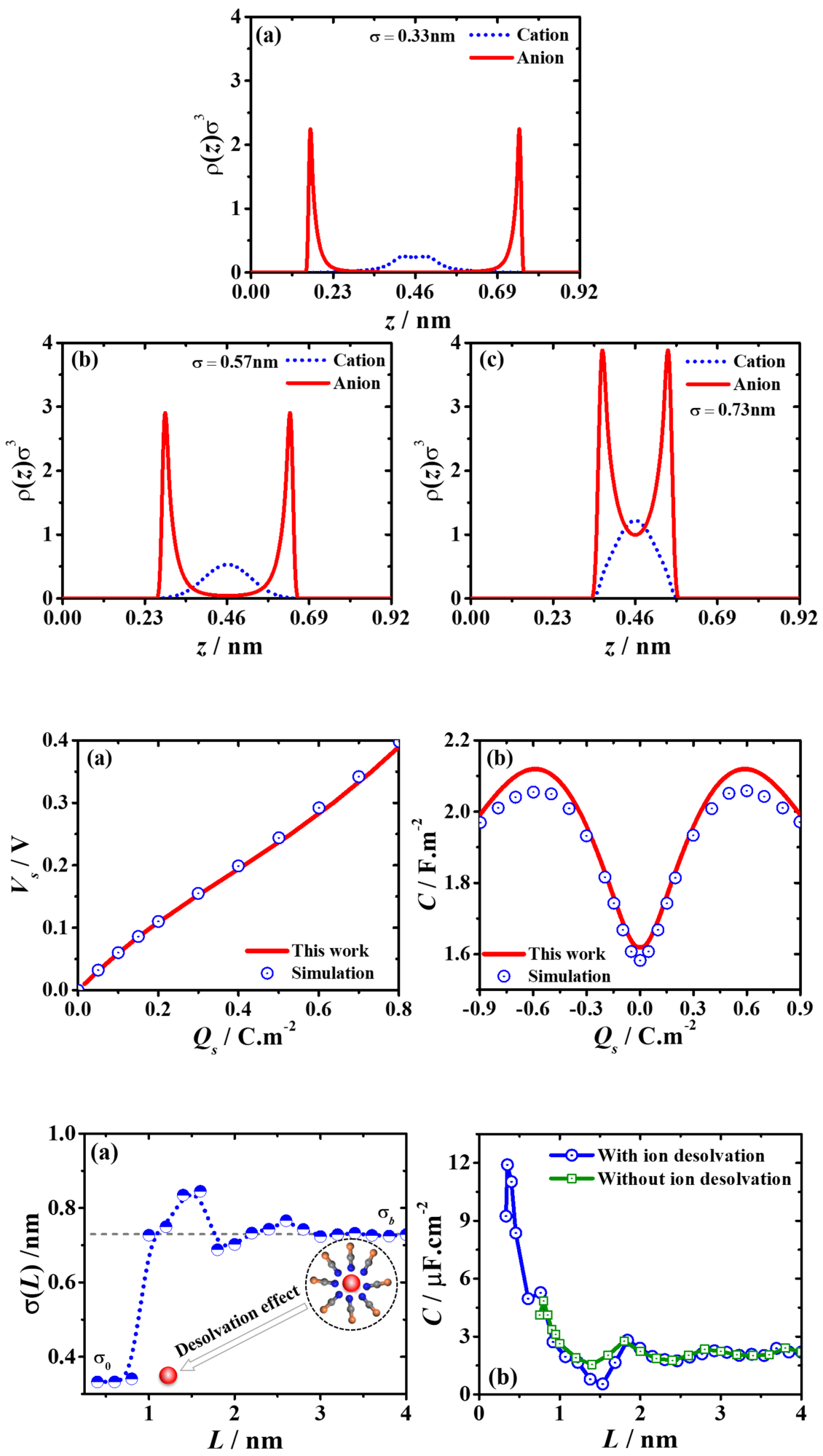


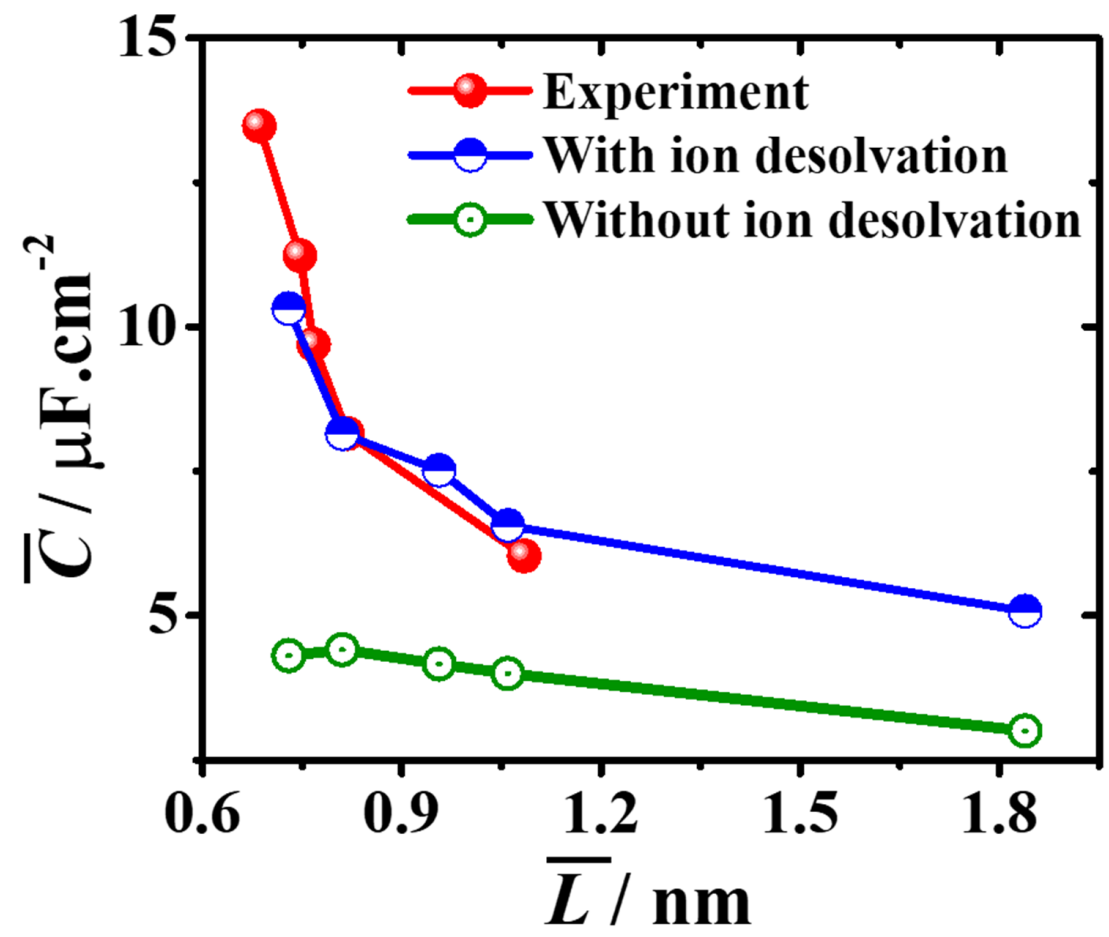

